\title{
The Stochastic Characteristics Method Applied to a Stochastic Schrödinger Equation
}

\author{
G. GUATTERI** AND H. LISEI*
}

\begin{abstract}
We study a stochastic Schrödinger equation using the method of stochastic characteristics, existence and uniqueness results are provided transforming the stochastic equation into a random family of deterministic equations. As a byproduct of the peculiar technique we easily prove that the solution defines a stochastic flow.
\end{abstract}

\section{IntRoduCtion}

In this paper we study the following equation:

$$
i d u(t, x)+\frac{\Delta}{2} u(t, x) d t-V(x) u(t, x) d t=\sum_{k=1}^{m} B_{k}(x) u(t, x) \circ d W^{k}(t)
$$

where the stochastic integral has to be interpreted as a Stratonovich integral $\left({ }^{1}\right)$. We will assume that the operators $B_{k}=i \sum_{j=1}^{d} b_{k j}(x) \partial_{j}$ are self adjoint in $L^{2}\left(\mathbb{R}^{d}\right)$ $=L^{2}\left(\mathbb{R}^{d} ; \mathbb{C}\right)$, then equation (1) turns out to be an example of the quantum filtering equation considered in [2] by Hudson and Parthasarathy in a completely different framework. Truman and Zastawniak consider in $[8,9]$ the particular case $k=1, d=1$ and $b \equiv 1$ : using a path integral approach they obtain a rigorous representation to the 1-dimensional stochastic Schrödinger equation in the whole space, see also [10] and the bibliography therein. Our aim is to find an existence and uniqueness result for Eq.(1), applying the method of stochastic characteristics, that consists in transforming the stochastic equation into a random family of equations, see for instance $[6,3,7]$ where the same method is developped in the parabolic case.

The present paper is organized as follows: in section 2 we introduce the equation for the stochastic characteristic and we illustrate some properties of the solution, in section 3 we prove an equivalence result between Eq.(1) and the random family of equations. In section 4 we provide an existence and uniqueness result for Eq.(1) starting from $u_{0} \in H^{2}\left(\mathbb{R}^{d} ; \mathbb{C}\right)$ at time $t=0$ and with $V \equiv 0$, finally in the last section we extend this result to the case with a potential $V \in C_{b}^{2}\left(\mathbb{R}^{d}\right)$.

\footnotetext{
${ }^{1}$ See the definition in Kunita,[4], for instance.
} 


\section{THE TRANSFORMATION}

We consider the following Cauchy problem:

$$
\left\{\begin{array}{l}
i d u(t, x)+\frac{1}{2} \Delta u(t, x) d t-V(x) u(t, x) d t= \\
i \sum_{k=1}^{m} \sum_{j=1}^{d} b_{k, j}(x) \partial_{j} u(t, x) \circ d W^{k}(t) \\
u(0)=u_{0},
\end{array}\right.
$$

where $V$ is a real function that acts on $u$ as a multiplication operator, and we will assume that $V \in C_{b}^{2}\left(\mathbb{R}^{d}\right)$ and that $W=\left(W^{1}, \ldots, W^{m}\right)$ is an $m$ dimensional Brownian motion. Let $\mathcal{F}_{t}$ be its natural filtration completed.

We denote by $\langle\cdot, \cdot\rangle$, without indexes, the scalar product in $\mathbb{R}^{d}$.

Hypothesis 2.1. We assume that:

$\left.A_{1}\right) b_{k, j} \in C^{4}\left(\mathbb{R}^{d} ; \mathbb{R}\right)$;

$\left.A_{2}\right)$ each $b_{k, i}$ is a linear function;

$\left.A_{3}\right)$ each vector function $b_{k}$, with $b_{k}=\left(b_{k, 1}, \ldots, b_{k, n}\right)^{*}$, is such that $\operatorname{div} b_{k}=0$.

We remark that, eventhought these assumptions are rather restrictive, we can deal with the cases $B=i \frac{d}{d x}$ in dimension 1 and $B=i\left(x_{2} \partial_{1}-x_{1} \partial_{2}\right)$ in dimension 3 , that corresponds respectively, to the momentum and the third component of the angular momentum and have some relevance in physics.

Definition 2.1. Let $u_{0} \in H^{1}\left(\mathbb{R}^{d}\right)$. We say that $u$ is a solution to (2) if $u \in$ $L_{a}^{2}\left(0, T ; H^{1}\left(\mathbb{R}^{d}\right)\right)\left({ }^{2}\right)$ and:

$$
\begin{aligned}
& \left(u(t)-u_{0}, v\right)_{L^{2}\left(\mathbb{R}^{d}\right)}=\frac{i}{2} \int_{0}^{t} H^{-1}\left(\mathbb{R}^{d}\right) \\
& -i \int_{0}^{t}(u(s), V v)_{L^{2}\left(\mathbb{R}^{d}\right)} d s+\int_{0}^{t} \sum_{k=1}^{m}\left(\left\langle b_{k}, \nabla u(s)\right\rangle, v\right\rangle_{H^{1}\left(\mathbb{R}^{d}\right)} d s
\end{aligned}
$$

for all $v \in H^{1}\left(\mathbb{R}^{d}\right)$,

We associate to (2) the following equation:

$$
\xi(t ; 0, x)=x-\sum_{k=1}^{m} \int_{0}^{t} b_{k}(\xi(s ; 0, x)) \circ d W^{k}(s)
$$

the solution $\xi(t, x)$ defines a stochastic flow of homeomorphisms $\left({ }^{3}\right)$, such that almost surely :

$$
\nu \leq \sup _{x \in \mathbb{R}^{d}}\left|D_{i \in[0, T]} \xi(t, x)\right| \leq C
$$

\footnotetext{
${ }^{2}$ We denote by $L_{a}^{2}\left(0, T ; H^{1}\left(\mathbb{R}^{d}\right)\right)$, the space of $L^{2}\left(\mathbb{R}^{d}\right)$ valued adapted processes $\phi$ such that $\mathbb{P}\left\{\|\phi(\omega)\|_{L^{2}\left(0, T ; H^{1}\left(\mathbb{R}^{d}\right)\right)}<\infty\right\}=1$

${ }^{3}$ The Stratonovich integral can be reduced to an Itô integral plus a bounded variation term under our regularity assumptions on the integrand.
} 
for any $i=1, \ldots, d$ and the constants $\nu$ and $C$ may depend on $\omega$. These estimates are consequence of the hypothesis on $b_{k, j}$, because:

(1) $\partial_{h} \xi$ solves an equation that does not depend on the $x$ variable;

(2) $\partial_{l h}^{2} \xi$ vanishes in every direction.

Similar properties has the inverse process $\eta(t, x)$, that is the solution to the following backward Stratonovich equation (see again [4]):

$$
d \eta(s ; t, x)=\sum_{k=1}^{m} b_{k}(\xi(s ; t, x)) \circ d W_{b}^{k}(s) .
$$

Note that under the condition $\operatorname{div} b_{k, j} \equiv 0$, the determinant of the Jacobian of $\xi(t ; 0, \cdot)$, denoted by $J_{0, t}$ is identically 1 , as it is the solution to:

$$
\begin{aligned}
J_{0, t}=1-\int_{0}^{t} \operatorname{div} b_{0}(\xi(r ; 0, x)) & J_{0, r}(x) d r \\
& -\sum_{k=1}^{m} \int_{0}^{t} \operatorname{div} b_{k}(\xi(r ; 0, x)) J_{0, r}(x) \circ d W_{k}(r)=1,
\end{aligned}
$$

where the final condition is given by the relation: $\eta(s ; t, \xi(t ; s, x))=x$.

We introduce the random operator $\Phi_{t}(\omega): L^{2}\left(\mathbb{R}^{d}\right) \mapsto L^{2}\left(\mathbb{R}^{d}\right)$ defined as

$$
\Phi_{t}(\omega) f(\cdot) \doteq f(\xi(t, \cdot, \omega)),
$$

from the properties described above it follows that this operator is a bounded linear and unitary operator on $L^{2}\left(\mathbb{R}^{d}\right)$.

Moreover its restriction to $D(\Delta)=H^{2}\left(\mathbb{R}^{d}\right)$ is still a bounded operator, actually $\Phi_{t}: H^{n}\left(\mathbb{R}^{d}\right) \rightarrow H^{n}\left(\mathbb{R}^{d}\right)$, for all integers $n$, is a bounded operator, as the second derivatives of $\xi$ vanish in every direction. We recall and summarize the properties of the family $\left\{\Phi_{t}\right\}_{t \in[0, T]}$ in the following proposition (we also use (4) and (6) to obtain these properties).

Proposition 2.1. The family $\left\{\Phi_{t}\right\}_{t \geq 0}$ enjoy the following properties:

(1) for each $t \in[0, T]$ and $\mathbb{P}$-a.s. the operator $\Phi_{t}$ defines an homeomorphism of $\mathbb{R}^{d}$

(2) $\Phi_{t} \in \mathcal{L}\left(L^{2}\left(\mathbb{R}^{d}\right)\right)$ and in $L^{2}\left(\mathbb{R}^{d}\right)$ it holds $\Phi_{t}^{*}=\Phi_{t}^{-1}$;

(3) $\Phi_{t} \in \mathcal{L}\left(H^{n}\left(\mathbb{R}^{d}\right)\right)$ for any integer $n \geq 1$.

\section{An Equivalence Result}

In this section we assume the existence of a weak solution of (2) and we compute the differential of $u(t, \xi(t, x))$. In order to do so, following [1] we compute an ItôWentzell formula, using a Galerkin approximation. In this case, as the problem is not of parabolic type, we do not have any smoothing property for the solution, therefore we must adapt the Itô-Wentzell formula for a process that does not have a version in the space of $C^{2}\left(\mathbb{R}^{d}\right)$. 
Before stating the result of this section we give the definition of solution for the random family of equations, consider the following problem:

$$
\left\{\begin{array}{rl}
i \frac{\partial}{\partial t} v(t, x, \omega)= & -\left(\Phi_{t}^{-1}\right)^{*}(\omega) \frac{\Delta}{2} \Phi_{t}^{-1}(\omega) v(t, x, \omega) \\
& +\Phi_{t}(\omega) V \Phi_{t}^{-1}(\omega) v(t, x, \omega) \\
v(0, x)=u_{0} &
\end{array} \quad t \in[0, T], x \in \mathbb{R}^{d}\right.
$$

Definition 3.1. $v(t, x, \omega)$ is a solution of $(7)$ if for all $\omega \in \Omega, v(\cdot, \cdot, \omega)$ is a weak solution of (7), in the sense that:

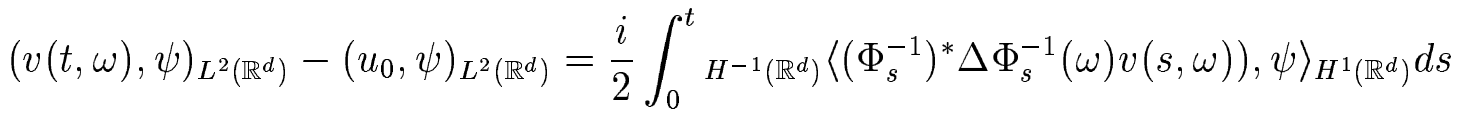

$$
\begin{aligned}
& -i \int_{0}^{t}\left(v(s, \omega), \Phi_{s} V \Phi_{s}^{-1}(\omega) \psi\right)_{L^{2}\left(\mathbb{R}^{d}\right)} d s
\end{aligned}
$$

for all $\psi \in H^{1}\left(\mathbb{R}^{d}\right)$. Moreover $v$ is a process in $L_{a}^{2}\left(0, T ; H^{1}\left(\mathbb{R}^{d}\right)\right)$.

Theorem 3.1. Let $u_{0} \in H^{1}\left(\mathbb{R}^{d}\right)$ then the stochastic differential equation:

$$
\left\{\begin{array}{l}
i d u(t)+\frac{\Delta}{2} u(t) d t-V u(t) d t= \\
\quad i \sum_{k=1}^{m}\left\langle b_{k}, \nabla u(t)\right\rangle \circ d W(t) \quad t \in[0, T], x \in \mathbb{R}^{d} \\
u(0, x)=u_{0}
\end{array}\right.
$$

is equivalent to the following evolution equation, depending on the parameter $\omega\left(^{4}\right)$ :

$$
\left\{\begin{array}{l}
i \frac{\partial}{\partial t} v(t, x, \omega)+\left(\Phi_{t}^{-1}\right)^{*}(\omega) \frac{\Delta}{2} \Phi_{t}^{-1}(\omega) v(t, x, \omega)= \\
\Phi_{t}(\omega) V \Phi_{t}^{-1}(\omega) v(t, x, \omega)=0 \quad t \in[0, T], x \in \mathbb{R}^{d} \\
v(0, x)=u_{0}
\end{array}\right.
$$

in the sense that if $u\left(t, x, u_{0}\right)$ is a solution of Eq.(2) then $v(t, x, \omega)=u(t, \xi(t, x), \omega)$ defines a solution for Eq.(9) and vice-versa.

Proof: For simplicity we will restrict to consider an one dimensional Wiener process. Let $e_{1}, \ldots, e_{n}, \ldots$ be a basis in $H^{1}\left(\mathbb{R}^{d}\right)$ that is orthonormal in $L^{2}\left(\mathbb{R}^{d}\right)$. We denote by $K_{n}=\operatorname{span}\left\{e_{1}, \ldots, e_{n}, \ldots\right\}$, and by $P_{n}$ the corresponding canonical projections of $L^{2}\left(\mathbb{R}^{d}\right)$ into $K_{n}$. In this way, for any $v \in L^{2}\left(\mathbb{R}^{d}\right)$

$$
P_{n} v(x)=v_{n}(x)=\sum_{j=1}^{n}\left(v, e_{j}\right)_{L^{2}\left(\mathbb{R}^{d}\right)} e_{j}(x) .
$$

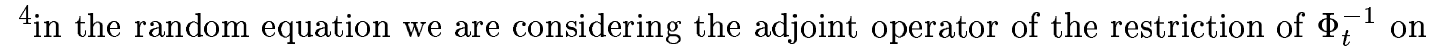
$H^{1}\left(\mathbb{R}^{d}\right)$
} 
Using the notation $u\left(t, \xi(t, x), u_{0}\right)=\Phi_{t}(\omega) u\left(t, x, u_{0}\right)$, we have

$$
\begin{aligned}
& \left(P_{n}\left(\Phi_{t} u_{n}\left(t, x, u_{0}\right)\right), v\right)_{L^{2}\left(\mathbb{R}^{d}\right)}= \\
& \sum_{j, k=1}^{n}\left(e_{j}, v\right)_{L^{2}\left(\mathbb{R}^{d}\right)}\left(u\left(t, x, u_{0}\right), e_{k}\right)_{L^{2}\left(\mathbb{R}^{d}\right)}\left(e_{k}, \Phi_{t}^{-1}(\omega) e_{j}\right)_{L^{2}\left(\mathbb{R}^{d}\right)}
\end{aligned}
$$

therefore:

$$
\begin{aligned}
& d\left(\left(P_{n}\left(\Phi_{t}(\omega) P_{n} u\left(t, x, u_{0}\right)\right), v\right)_{L^{2}\left(\mathbb{R}^{d}\right)}\right. \\
& =d \sum_{j, k=1}^{n}\left(e_{j}, v\right)_{L^{2}\left(\mathbb{R}^{d}\right)}\left(u\left(t, x, u_{0}\right), e_{k}\right)_{L^{2}\left(\mathbb{R}^{d}\right)}\left(e_{k}, \Phi_{t}^{-1}(\omega) e_{j}\right)_{L^{2}\left(\mathbb{R}^{d}\right)} \\
& =\sum_{j, k=1}^{n}\left(e_{j}, v\right)_{L^{2}\left(\mathbb{R}^{d}\right)}\left(d u\left(t, x, u_{0}\right), e_{k}\right)_{L^{2}\left(\mathbb{R}^{d}\right)}\left(e_{k}, \Phi_{t}^{-1}(\omega) e_{j}\right)_{L^{2}\left(\mathbb{R}^{d}\right)} \\
& +\sum_{j, k=1}^{n}\left(e_{j}, v\right)_{L^{2}\left(\mathbb{R}^{d}\right)}\left(u\left(t, x, u_{0}\right), e_{k}\right)_{L^{2}\left(\mathbb{R}^{d}\right)}\left(d \Phi_{t}(\omega) e_{k}, e_{j}\right)_{L^{2}\left(\mathbb{R}^{d}\right)} \\
& =\sum_{j, k=1}^{n}\left(e_{j}, v\right)_{L^{2}\left(\mathbb{R}^{d}\right)}\left(b(x) u_{x}\left(t, x, u_{0}\right) \circ d W(t), e_{k}\right)_{L^{2}\left(\mathbb{R}^{d}\right)}\left(e_{k}, \Phi_{t}^{-1}(\omega) e_{j}\right)_{L^{2}\left(\mathbb{R}^{d}\right)} \\
& +i \sum_{j, k=1}^{n}\left(e_{j}, v\right)_{L^{2}\left(\mathbb{R}^{d}\right) H^{-1}\left(\mathbb{R}^{d}\right)}\left\langle\frac{\Delta}{2} u\left(t, x, u_{0}\right) d t, e_{k}\right\rangle_{H^{1}\left(\mathbb{R}^{d}\right)}\left(e_{k}, \Phi_{t}^{-1}(\omega) e_{j}\right)_{L^{2}\left(\mathbb{R}^{d}\right)} \\
& -i \sum_{j, k=1}^{n}\left(e_{j}, v\right)_{L^{2}\left(\mathbb{R}^{d}\right)}\left(V u\left(t, x, u_{0}\right), e_{k}\right)_{L^{2}\left(\mathbb{R}^{d}\right)}\left(e_{k}, \Phi_{t}^{-1}(\omega) e_{j}\right)_{L^{2}\left(\mathbb{R}^{d}\right)} \\
& +\sum_{j, k=1}^{n}\left(e_{j}, v\right)_{L^{2}\left(\mathbb{R}^{d}\right)}\left(u\left(t, x, u_{0}\right), e_{k}\right)_{L^{2}\left(\mathbb{R}^{d}\right)}\left(d \Phi_{t}(\omega) e_{k}, e_{j}\right)_{L^{2}\left(\mathbb{R}^{d}\right)} .
\end{aligned}
$$

By the Itô formula we obtain

$$
d_{s} \Phi_{t}(\omega) e_{k}=d e_{k}(\xi(t, \cdot))=\nabla e_{k}(\xi(t, \cdot)) d_{s} \xi(t, \cdot)=-\left\langle\nabla e_{k}(\xi(t, \cdot)), b(\xi(t, \cdot)\rangle \circ d W(t) .\right.
$$

Therefore, knowing that $\operatorname{div} b=0$ :

$$
\begin{aligned}
& \sum_{j, k=1}^{n} e_{j}\left(u\left(t, u_{0}\right), e_{k}\right)_{L^{2}\left(\mathbb{R}^{d}\right)}\left(d \Phi_{t}(\omega) e_{k}, e_{j}\right)_{L^{2}\left(\mathbb{R}^{d}\right)} \\
& =-\sum_{j, k=1}^{n} e_{j}\left(u\left(t, u_{0}\right), e_{k}\right)_{L^{2}\left(\mathbb{R}^{d}\right)}\left(\left\langle\nabla e_{k}(\xi(t)), b(\xi(t))\right\rangle \circ d W(t), e_{j}\right)_{L^{2}\left(\mathbb{R}^{d}\right)} \\
& =\sum_{j, k=1}^{n} e_{j}\left(u\left(t, u_{0}\right), e_{k}\right)_{L^{2}\left(\mathbb{R}^{d}\right)}\left(e_{k},\left\langle b, \nabla\left(\Phi_{t}^{-1}(\omega) e_{j}\right)\right\rangle \circ d W(t)\right)_{L^{2}\left(\mathbb{R}^{d}\right)}
\end{aligned}
$$


and substituting the latter formula in the former one, we get

$$
\begin{aligned}
& d\left(\left(P_{n}\left(\Phi_{t}(\omega) P_{n} u\left(t, u_{0}\right)\right)\right), v\right)_{L^{2}\left(\mathbb{R}^{d}\right)} \\
= & d \sum_{j, k=1}^{n}\left(e_{j}, v\right)_{L^{2}\left(\mathbb{R}^{d}\right)}\left(u\left(t, u_{0}\right), e_{k}\right)_{L^{2}\left(\mathbb{R}^{d}\right)}\left(e_{k}, \Phi_{t}^{-1}(\omega) e_{j}\right)_{L^{2}\left(\mathbb{R}^{d}\right)} \\
= & \left.\sum_{j, k=1}^{n}\left(e_{j}, v\right)_{L^{2}\left(\mathbb{R}^{d}\right)}\left\langle b, \nabla u\left(t, u_{0}\right)\right\rangle \circ d W(t), e_{k}\right)_{L^{2}\left(\mathbb{R}^{d}\right)}\left(e_{k}, \Phi_{t}^{-1}(\omega)\left(e_{j}\right)_{L^{2}\left(\mathbb{R}^{d}\right)}\right. \\
& +i \sum_{j, k=1}^{n}\left(e_{j}, v\right)_{L^{2}\left(\mathbb{R}^{d}\right) H^{-1}\left(\mathbb{R}^{d}\right)}\left\langle\frac{\Delta}{2} u\left(t, u_{0}\right) d t, e_{k}\right\rangle_{H^{1}\left(\mathbb{R}^{d}\right)}\left(e_{k}, \Phi_{t}^{-1}(\omega)\left(e_{j}\right)_{L^{2}\left(\mathbb{R}^{d}\right)}\right. \\
& \left.+\sum_{j, k=1}^{n}\left(e_{j}, v\right)_{L^{2}\left(\mathbb{R}^{d}\right)}\left(u\left(t, u_{0}\right), e_{k}\right)_{L^{2}\left(\mathbb{R}^{d}\right)}\left(e_{k},\left\langle b, \nabla\left(\Phi_{t}^{-1}(\omega) e_{j}\right)\right\rangle d W(t)\right)_{L^{2}\left(\mathbb{R}^{d}\right)}\right) \\
& -i \sum_{j, k=1}^{n}\left(e_{j}, v\right)_{L^{2}\left(\mathbb{R}^{d}\right)}\left(V u\left(t, u_{0}\right), e_{k}\right)_{L^{2}\left(\mathbb{R}^{d}\right)}\left(e_{k}, \Phi_{t}(\omega)^{-1} e_{j}\right)_{L^{2}\left(\mathbb{R}^{d}\right)} \\
= & \sum_{j, k=1}^{n}\left(e_{j}, v\right)_{L^{2}\left(\mathbb{R}^{d}\right)}\left(\left\langle b, \nabla u\left(t, u_{0}\right)\right\rangle \circ d W(t), e_{k}\right)_{L^{2}\left(\mathbb{R}^{d}\right)}\left(e_{k}, \Phi_{t}^{-1}(\omega) e_{j}\right)_{L^{2}\left(\mathbb{R}^{d}\right)} \\
& +\frac{i}{2} \sum_{j, k=1}^{n}\left(e_{j}, v\right)_{L^{2}\left(\mathbb{R}^{d}\right) H{ }^{-1}\left(\mathbb{R}^{d}\right)}\left\langle\Delta u\left(t, u_{0}\right) d t, e_{k}\right\rangle_{H^{1}\left(\mathbb{R}^{d}\right)}\left(e_{k}, \Phi_{t}^{-1}(\omega) e_{j}\right)_{L^{2}\left(\mathbb{R}^{d}\right)} \\
& -\sum_{j, k=1}^{n}\left(e_{j}, v\right)_{L^{2}\left(\mathbb{R}^{d}\right)}\left(\left\langle b, \nabla u\left(t, u_{0}\right)\right\rangle \circ d W(t), e_{k}\right)_{L^{2}\left(\mathbb{R}^{d}\right)}\left(e_{k}, \Phi_{t}^{-1}(\omega) e_{j}\right)_{L^{2}\left(\mathbb{R}^{d}\right)} \\
& -i \sum_{j, k=1}^{n}\left(e_{j}, v\right)_{L^{2}\left(\mathbb{R}^{d}\right)}\left(V u\left(t, u_{0}\right), e_{k}\right)_{L^{2}\left(\mathbb{R}^{d}\right)}\left(e_{k}, \Phi_{t}^{-1}(\omega) e_{j}\right)
\end{aligned}
$$

We write it in the integral form:

$$
\begin{aligned}
& \left(P_{n}\left(\Phi_{t}(\omega) P_{n} u\left(t, u_{0}\right)\right), v\right)_{L^{2}\left(\mathbb{R}^{d}\right)}=\left(P_{n} u_{0}, v\right) \\
& +\frac{i}{2} \int_{0}^{t} \sum_{j, k=1}^{n}\left(e_{j}, v\right)_{L^{2}\left(\mathbb{R}^{d}\right) H^{-1}\left(\mathbb{R}^{d}\right)}\left\langle\Delta u\left(s, u_{0}\right), e_{k}\right\rangle_{H^{1}\left(\mathbb{R}^{d}\right)}\left(e_{k}, \Phi_{s}^{-1}(\omega) e_{j}\right)_{L^{2}\left(\mathbb{R}^{d}\right)} d s \\
& -i \int_{0}^{t}\left(P_{n} \Phi_{s} P_{n} V u\left(s, u_{0}\right), v\right)_{L^{2}\left(\mathbb{R}^{d}\right)} d s .
\end{aligned}
$$

The left hand side converges to $\left(\Phi_{t}(\omega) u\left(t, u_{0}\right), v\right)_{\left.L^{2}\left(\mathbb{R}^{d}\right)\right)}$ as $n$ goes to infinity, as $P_{n} \rightarrow I$ in $L^{2}\left(\mathbb{R}^{d}\right)$.

For the same reason also the first and the last members at the right hand side converge respectively to $\left(u_{0}, v\right)_{L^{2}\left(\mathbb{R}^{d}\right)}$ and to $\int_{0}^{t}\left(\Phi_{s} V u\left(s, u_{0}\right), v\right)_{L^{2}\left(\mathbb{R}^{d}\right)} d s$. We rewrite 
the second member at the right hand side:

$$
\begin{aligned}
& \int_{0}^{t} \sum_{j, k=1}^{n}\left(e_{j}, v\right)_{L^{2}\left(\mathbb{R}^{d}\right) H^{-1}\left(\mathbb{R}^{d}\right)}\left\langle\frac{i \Delta}{2} u\left(s, u_{0}\right), e_{k}\right\rangle_{H^{1}\left(\mathbb{R}^{d}\right)}\left(e_{k}, \Phi_{s}^{-1}(\omega) e_{j}\right)_{L^{2}\left(\mathbb{R}^{d}\right)} d s \\
& =\int_{0}^{t} H^{-1\left(\mathbb{R}^{d}\right)}\left\langle\frac{i \Delta}{2} u\left(s, u_{0}\right), P_{n} \Phi_{s}^{-1} P_{n} v\right\rangle_{H^{1}\left(\mathbb{R}^{d}\right)} d s,
\end{aligned}
$$

where $\Phi_{t}$ is the restriction of $\Phi_{t}$ to $H^{1}\left(\mathbb{R}^{d}\right)$. Passing to the limit, we obtain that the latter integral converges to:

$$
\frac{i}{2} \int_{0}^{t} H^{-1}\left(\mathbb{R}^{d}\right)\left\langle\left(\Phi_{s}^{-1}\right)^{*} \Delta u\left(s, u_{0}\right) d s, v\right\rangle_{H^{1}\left(\mathbb{R}^{d}\right)}
$$

all these convergences hold for almost all $\omega \in \Omega$.

Finally, denoting by $v\left(s, x, u_{0}\right)=\Phi_{s}(\omega) u\left(s, x, u_{0}\right)$ we have that, for all $v \in H^{1}\left(\mathbb{R}^{d}\right)$ :

$$
\begin{aligned}
& \left(v\left(t, u_{0}\right), v\right)_{L^{2}\left(\mathbb{R}^{d}\right)}=\left(u_{0}, v\right)_{L^{2}\left(\mathbb{R}^{d}\right)} \\
& +\frac{i}{2} \int_{0}^{t} H^{-1}\left(\mathbb{R}^{d}\right)\left\langle\left(\Phi_{s}^{-1}\right)^{*} \Delta \Phi_{s}^{-1}(\omega) v\left(s, u_{0}\right), v\right\rangle_{H^{1}\left(\mathbb{R}^{d}\right)} d s \\
& -i \int_{0}^{t}\left(v\left(s, u_{0}\right), \Phi_{s} V \Phi_{s}^{-1}(\omega) v\right)_{L^{2}\left(\mathbb{R}^{d}\right)} d s .
\end{aligned}
$$

The converse implication follows exactly in the same way.

Remark 3.1. Note that the operator $\frac{1}{2}\left(\Phi_{t}^{-1}\right)^{*} \Delta \Phi_{t}^{-1}-\Phi_{t} V \Phi_{t}^{-1}$ is still self adjoint in the space $L^{2}\left(\mathbb{R}^{d}\right)$ and so, by Stone theorem (see for instance [5]), the operator $i\left[\frac{1}{2}\left(\Phi_{t}^{-1}\right)^{*} \Delta \Phi_{t}^{-1}-\Phi_{t} V \Phi_{t}^{-1}\right]$ generates a unitary group at each $t$ fixed.

\section{EXISTENCE RESULT FOR THE RANDOM EQUATION}

4.1. The case with zero potential. Now we look for a solution of the random family of deterministic equations, starting with the case $V \equiv 0$.

In most of the section we will omit the $\omega$ dependence as we will consider the equation pathwise. We rewrite problem (9) in abstract notation:

$$
\left\{\begin{array}{l}
u^{\prime}(t)=A(t) u(t) \\
u(0)=u_{0}
\end{array}\right.
$$

where $A(t)=i \frac{1}{2}\left(\Phi_{t}^{-1}\right)^{*} \Delta \Phi_{t}^{-1}$. Following [5], we recall some results that we will use in the sequel:

Theorem 4.1 ([5], Theorem 2.2). For $t \in[0, T]$ let $A(t)$ be the infinitesimal generator of a $C_{0}$ semigroup $T_{t}(s)$ on the Banach space $X$. The family of generators 
$\{A(t)\}_{t \in[0, T]}$ is stable if and only if there are constants $M \geq 1$ and $\omega$ such that $\rho(A(t)) \supset] \omega, \infty]$ for $t \in[0, T]$ and either one of the following conditions is satisfied:

$$
\left\|\prod_{j=1}^{k} R\left(\lambda_{j}, A\left(t_{j}\right)\right)\right\|_{\mathcal{L}(X)} \leq M \prod_{j=1}^{k}\left(\lambda_{j}-\omega\right)^{-1} \quad \forall \lambda_{j}>\omega
$$

for any finite sequence $0 \leq t_{1} \leq t_{2} \leq \cdots \leq t_{k} \leq T, k=1,2, \ldots$, or

$$
\left\|\prod_{j=1}^{k} T_{t_{j}}\left(s_{j}\right)\right\|_{\mathcal{L}(X)} \leq M \exp \left\{\omega \sum_{j=1}^{k} s_{j}\right\} \forall \lambda_{j}>\omega
$$

for any finite sequence $0 \leq t_{1} \leq t_{2} \leq \cdots \leq t_{k} \leq T, k=1,2, \ldots$.

If $T_{t_{j}}\left(s_{j}\right)$ are contraction semigroups for every $t_{j} \in[0, T]$, then estimate (14) is verified with $M=1$ and $\omega=0$.

We recall the notion of admissible subspace of $X$ :

Definition 4.1. There are given a $C_{0}$-semigroup $T(t)$ and its infinitesimal generator A. A subspace $Y$ of $X$ is called A-admissible if it is an invariant subspace of $\{T(t), t \geq 0\}$, and the restriction of $T(t)$ to $Y$ is a $C_{0}$-semigroup in $Y$.

Finally we recall the existence result for the evolution system; we are given the following assumptions:

$\left.H_{1}\right)\{A(t)\}_{t \in[0, T]}$ is a stable family with stability constants $M, \omega$,

$H_{2}$ ) There exists a dense subspace $Y \subset X$ s.t. $Y$ is $A(t)$-admissible for every $t \in[0, T]$ and the family $\{\tilde{A}(t)\}_{t \in[0, T]}$ of parts $\tilde{A}(t)\left(^{5}\right)$ of $A(t)$ in $Y$, is a stable family in $Y$ with stability constants $\tilde{M}, \tilde{\omega}$,

$\left.H_{3}\right)$ for $t \in[0, T], D(A(t)) \supset Y, A(t)$ is a bounded operator from $Y$ into $X$ and $t \rightarrow A(t)$ is continuous in the $\mathcal{L}(Y, X)$ norm $\|\cdot\|_{Y \rightarrow X}$.

Theorem 4.2 ([5], Theorem 3.1). Let $A(t), 0 \leq t \leq T$, be the infinitesimal generator of a $C_{0}$ semigroup $\{T(s), s \geq 0\}$, on $X$. If the family $\{A(t)\}_{t \in[0, T]}$ satisfies the conditions $H_{1}-H_{3}$ then there exists a unique evolution system $U(t, s)$, $0 \leq s \leq t \leq T$, in $X$ satisfying:

$$
\begin{array}{cc}
\|U(t, s)\|_{\mathcal{L}(X)} \leq M \exp \{\omega(t-s)\} & 0 \leq s \leq t \leq T \\
\left.\frac{\partial^{+}}{\partial t} U(t, s) v\right|_{t=s}=A(s) v & v \in Y, 0 \leq s \leq t \leq T \\
\frac{\partial}{\partial s} U(t, s) v=-U(t, s) A(s) v & v \in Y, 0 \leq s \leq t \leq T .
\end{array}
$$

We will use also the following:

\footnotetext{
${ }^{5}$ The part of $A$ in $Y$ is defined as the restriction of $A$ to $Y, \tilde{A}: D(\tilde{A})=\{z \in D(A) \cap Y\} \rightarrow Y$, and $\tilde{A} y=A y$ for all $y \in D(A)$.
} 
Theorem 4.3 ([5], Theorem 4.3). Let $A(t), 0 \leq t \leq T$, satisfy the conditions of the previous theorem and let $U(t, s)$ be the evolution system defined above. If

$$
U(t, s) Y \subset Y \quad 0 \leq s \leq t \leq T
$$

and

$$
\text { for } v \in Y \quad U(t, s) v \text { is continuous in } Y \text { for } 0 \leq s \leq t \leq T \text {, }
$$

then for every $v \in Y, U(t, s) v$ is the unique $Y$-valued solution of the initial value problem:

$$
\left\{\begin{array}{l}
u^{\prime}(t)=A(t) u(t) \\
u(s)=v
\end{array}\right.
$$

By $Y$-valued solution we mean the following:

Definition 4.2. A function $u \in C([0, T] ; Y)$ is a $Y$-valued solution of $(20)$ if $\left.\left.u \in C^{1}(] 0, T\right] ; X\right)$ and $(20)$ is satisfied by $u$ in $X$.

We apply this abstract theory to problem (9) derived in the previous section. Actually as we follow a semigroup approach we are lead to consider more regular solutions, i.e. with value in the domain of the operator $A(t)=\frac{i}{2}\left(\Phi(t)^{-1}\right)^{*} \Delta \Phi(t)^{-1}$. This domain is constant in time and equivalent to $H^{2}\left(\mathbb{R}^{d}\right)$ hence, recalling that $\Phi_{t}$ is unitary in $L^{2}\left(\mathbb{R}^{d}\right)$, we rewrite $A(t)$ as $A(t) \doteq \frac{i}{2} \Phi(t) \Delta \Phi(t)^{-1}$. As first result we prove:

Theorem 4.4. The family of operators $\{A(t), t \in[0, T]\}$ defined above generates a unique evolution system $U(t, s)$ such that:

$$
\|U(t, s)\|_{\mathcal{L}(X)} \leq C .
$$

Proof: We will show that the hypotheses $H_{1}-H_{3}$ are verified in our situation. We will denote by $T_{t_{0}}(t)$ the group generated by $A\left(t_{0}\right)$, from the definition of $C_{0^{-}}$ semigroup it follows that $T_{t_{0}}(t)=\Phi\left(t_{0}\right) e^{\frac{i}{2} \Delta t} \Phi\left(t_{0}\right)^{-1}$. Hypothesis $H_{1}$ corresponds to the stability for the family of $\{A(t)\}_{t \in[0, T]}$ and can be replaced by the two equivalent conditions of theorem 4.1, that are easier to verify in this case. First, by remark 3.1, the condition:

$$
\Pi_{i+1}^{k}\left\|T_{t_{i}}\left(s_{i}\right)\right\|_{\mathcal{L}(X)} \leq 1 \quad s_{i} \geq 0
$$

is fulfilled (with constant $M=1$ and $\omega=0$ ) for any finite sequence $0 \leq t_{1} \leq t_{2} \leq$ $\cdots \leq t_{k} \leq T, k \in \mathbf{N}$. The other condition, $\left.\rho(A(t)) \supset\right] 0,+\infty[$ follows from the observation that:

$$
(\lambda I-A(t))^{-1}=\Phi(t)^{-1}(\lambda I-i \Delta)^{-1} \Phi(t)
$$

and then $\lambda \in \rho(i \Delta) \leftrightarrow \lambda \in \rho(A(t))$. Then $\rho(A(t))=\rho(i \Delta) \supset] 0, \infty[$.

Hypothesis $H_{2}$ requires that there exists a subset $Y$ of $L^{2}\left(\mathbb{R}^{d}\right)$ such that for $t \in$ $[0, T], Y$ is $A(t)$-admissible and the family $\{\tilde{A}(t)\}_{t \in[0, T]}$ of the parts of $A(t)$ is a 
stable family in $Y$ with some uniform constants $\tilde{M}$ and $\tilde{\omega}$. In our case we will take as $Y$ the constant domain $H^{2}\left(\mathbb{R}^{d}\right)$ of each $A(t)$. Since the first derivatives of the $\eta$ and $\xi$ do not depend on $x$, the operator $i A(t)$ at every $t$ is a second order operator in divergence form whose matrix of coefficients is constant and symmetric. Therefore $i A(t)$ is still selfadjoint on $H^{2}\left(\mathbb{R}^{d}\right)$ so $A(t)$ generates a unitary group on $H^{2}\left(\mathbb{R}^{d}\right)$ at every fixed $t$; this conclude the proof of the admissibility. Moreover we can prove the following estimate

$$
\left\|T_{t_{0}}(t)_{\left.\right|_{H^{2}\left(\mathbb{R}^{d}\right)}} u\right\|_{H^{2}\left(\mathbb{R}^{d}\right)} \leq\|u\|_{H^{2}\left(\mathbb{R}^{d}\right)},
$$

where $\tilde{M}=1$ and $\tilde{\omega}=0$. This verifies the stability condition for the family of the parts $\tilde{A}(t)$, thus $H_{2}$ is satisfied.

Hypothesis $H_{3}$ requires that $t \mapsto A(t)$ is continuous in the norm of the space $\mathcal{L}\left(H^{2}\left(\mathbb{R}^{d}\right), L^{2}\left(\mathbb{R}^{d}\right)\right)$, the space of bounded and linear operators from $H^{2}\left(\mathbb{R}^{d}\right)$ into $L^{2}\left(\mathbb{R}^{d}\right)$, but this follows from the properties of $\Phi(t)$ and of the Laplacian. Then we can define the evolution system $U(t, s)$.

Our intention is to find a representation for the solution of (12) in terms of the evolution system $U(t, s)$ generated by $A(t)$. In order to apply theorem 4.3 we still have to prove that $U(t, 0)\left(H^{2}\left(\mathbb{R}^{d}\right)\right) \subset H^{2}\left(\mathbb{R}^{d}\right)$ and that $t \rightarrow U(t, 0) v$ is continuous in $H^{2}\left(\mathbb{R}^{d}\right)$ for $0 \leq t \leq T$. We collect these results in following lemma:

Lemma 4.1. The domain $H^{2}\left(\mathbb{R}^{d}\right)$ is invariant under the action of the evolution system $U(t, s)$ and $t \rightarrow U(t, 0) v$ is a continuous mapping in $H^{2}\left(\mathbb{R}^{d}\right)$ for any $y \in$ $H^{2}\left(\mathbb{R}^{d}\right)$.

Proof: Let us take now as $X=H^{2}\left(\mathbb{R}^{2}\right)$ and as $Y=H^{4}\left(\mathbb{R}^{2}\right)$, the constant domain of $\{i A(t), t \in[0, T]\}$ in $X$. Since $i A(t)$ is also self-adjoint in $H^{4}\left(\mathbb{R}^{2}\right)$ at each $t \in[0, T]$, then theorem 4.4 holds also in this case. Therefore $\|U(t, s) v\|_{H^{2}\left(\mathbb{R}^{2}\right)} \leq C\|v\|_{H^{2}\left(\mathbb{R}^{2}\right)}$, for every $v \in H^{2}\left(\mathbb{R}^{2}\right)$, by the property of the evolution system $U(t, s)$. This concludes the proof.

Finally we can state the following existence theorem:

Theorem 4.5. Problem (9), with $V \equiv 0$, has a unique $H^{2}\left(\mathbb{R}^{d}\right)$-valued solution $v(t, \cdot, \omega)=U(t, 0, \omega) u_{0}$ for all $u_{0} \in H^{2}\left(\mathbb{R}^{d}\right)$ and almost all $\omega \in \Omega$. $U(t, s, \omega)$ is the evolution system defined in theorem 4.4 that is unitary, i.e. $\left\|U(t, s, \omega) u_{0}\right\|_{L^{2}\left(\mathbb{R}^{d}\right)}=$ $\left\|u_{0}\right\|_{L^{2}\left(\mathbb{R}^{d}\right)}$ for all $u_{0} \in H^{2}\left(\mathbb{R}^{d}\right)$ and for almost all $\omega$.

This solution $\left.\left.v \in C\left([0, T] ; H^{2}\left(\mathbb{R}^{d}\right)\right) \cap C^{1}(] 0, T\right] ; L^{2}\left(\mathbb{R}^{d}\right)\right)$ is $\mathcal{F}_{t}$ adapted.

Proof: The existence, uniqueness and regularity results for the solution are consequence of the previous results. Finally, if one evaluate the energy of the solution 
$v$ of (12):

$$
\operatorname{Re}\left[\left(v^{\prime}(t), v(t)\right)_{L^{2}\left(\mathbb{R}^{d}\right)}-i\left(\Phi_{t} \frac{\Delta}{2} \Phi_{t}^{-1} v(t), v(t)\right)_{L^{2}\left(\mathbb{R}^{d}\right)}\right]=\frac{1}{2} \frac{d}{d t}|v(t)|_{L^{2}\left(\mathbb{R}^{d}\right)}^{2}=0
$$

this implies, passing to the real part of the equation, that

$$
|u(t, \omega)|_{L^{2}\left(\mathbb{R}^{d}\right)}=\left|u_{0}\right|_{L^{2}\left(\mathbb{R}^{d}\right)} .
$$

At this level of regularity this solution is in particular a solution of problem (9) in the sense of definition 3.1 , so $\Phi_{t}^{-1} v$ defines a solution for problem (2). The adaptedness of $v$ is a consequence of the peculiar construction of the solution: $U(t, 0) u_{0}$ is obtained as a limit of unitary groups depending only on $s \leq t$. In this way, starting from an adapted initial datum the solution remains always adapted.

As a byproduct of the equivalence result, we have:

Corollary 4.1. The unique solution $u(t, x)=\Phi_{t}^{-1}(v)(t, x)$ is a stochastic flow.

Proof: Let $u\left(t ; s, u_{0}\right)$ be the solution to $(2)$ starting at time $s>0$. From the property of backward flow $\eta, \eta(s ; t, x)=\eta(s ; r, \eta(t, x))$ we get that there exists the random evolution operator $G(t, s) u_{0}=u\left(t ; s, u_{0}\right)=\Phi_{t}^{-1} U(t, s) u_{0}$.

Therefore as both $\Phi_{t}^{-1}$ and $U(t, s)$ are unitary we have that

$$
\left\|G(t, s) u_{0}\right\|_{L^{2}\left(\mathbb{R}^{d}\right)}=\left\|\Phi_{t}^{-1} U(t, s) u_{0}\right\|_{L^{2}\left(\mathbb{R}^{d}\right)}=\left\|u_{0}\right\|_{L^{2}\left(\mathbb{R}^{d}\right)} .
$$

4.2. The case with bounded potentials. In this section we add the potential $V$ assuming that it is a real function in $C_{b}^{2}\left(\mathbb{R}^{d}\right)$, then the family of deterministic equations becomes, written in abstract form:

$$
\left\{\begin{array}{l}
u^{\prime}(t)=A(t) u(t)-i V(t) u(t) \quad 0 \leq t \leq T \\
u(0)=u_{0}
\end{array}\right.
$$

where $V(t)=\Phi_{t} V \Phi_{t}^{-1}$. Let us notice first that $\|V(t)\|_{C_{b}^{2}\left(\mathbb{R}^{d}\right)} \leq C\|V\|_{C_{b}^{2}\left(\mathbb{R}^{d}\right)}$. We treat the potential as a perturbation of the operator $A$, and we can prove the following:

Theorem 4.6. If $V \in C_{b}^{2}\left(\mathbb{R}^{d}\right)$, then problem (9) has a solution with the same properties as the solution found in theorem 4.5.

Proof: The function $V$ is a bounded self adjoint operator from $L^{2}\left(\mathbb{R}^{d}\right)$ into itself and a bounded operator in $H^{2}\left(\mathbb{R}^{d}\right)$. Hence it is a standard fact that the sum $i A(t)+V(t)$ is still self- adjoint in $L^{2}\left(\mathbb{R}^{d}\right)$ for every $t \in[0, T]$, therefore $A(t)-i V(t)$ generates a unitary group in $L^{2}\left(\mathbb{R}^{d}\right)$, and hypothesis $H_{1}$ is verified. As the domain is still $H^{2}\left(\mathbb{R}^{d}\right)$ we choose again it as the $[A(t)-i V(t)]$ - admissible subspace, required 
in hypothesis $\mathrm{H}_{2}$.

We need to show that the part of $A(t)-i V(t)$ in $H^{2}\left(\mathbb{R}^{d}\right)$, that is the sum of the parts $\tilde{A}(t)$ and $-i \tilde{V}(t)$, is still an infinitesimal generator of a $C_{0}$ semigroup. For this purpose we will apply the Hille-Yoshida Theorem, see [5, Theorem 5.3]. We verify the two conditions required to prove the theorem. The first is immediately verified, as $i \tilde{V}(t)$ is continuous in $H^{2}\left(\mathbb{R}^{d}\right)$ and $\tilde{A}(t)$ is a generator in $H^{2}\left(\mathbb{R}^{d}\right)$ hence the sum $\tilde{A}(t)-i \tilde{V}(t)$ is a closed operator in $H^{2}\left(\mathbb{R}^{d}\right)$ for all $t \in[0, T]$.

Then we need to show that there exists a $\lambda_{0}$ such that the ray $\{\lambda \in \mathbf{C}, \operatorname{Im} \lambda=$ $\left.0, \lambda>\lambda_{0}\right\}$ is contained in the resolvent set $\rho(\tilde{A}(t))$. Moreover we have to prove an estimate for the resolvent $R(\lambda ; A(t)-i V(t))$ of the following form:

$$
\left\|R(\lambda ; \tilde{A}(t)-i \tilde{V}(t))^{n}\right\|_{H^{2}\left(\mathbb{R}^{d}\right)} \leq \frac{M}{\left(\lambda-\lambda_{0}\right)^{n}} \quad \forall \lambda>\lambda_{0} .
$$

Since $\tilde{A}(t)$ is the generator of a $C_{0}$ semigroup with $\tilde{\omega}=0$ and $\tilde{M}=1$, then

$$
\|R(\lambda ; \tilde{A}(t))\|_{H^{2}\left(\mathbb{R}^{d}\right)} \leq \frac{1}{\lambda^{n}}, \quad \forall \lambda>0
$$

in our case we have that:

$$
\begin{array}{r}
\left\|R(\lambda ; \tilde{A}(t)-i \tilde{V}(t))^{n}\right\|_{H^{2}\left(\mathbb{R}^{d}\right)}=\left\|R(\lambda ; \tilde{A}(t))^{n}(I-i \tilde{V}(t) R(\lambda ; \tilde{A}(t)))^{-n}\right\|_{H^{2}\left(\mathbb{R}^{d}\right)} \\
\leq \frac{1}{\lambda^{n}} \leq \frac{1}{\left(\lambda-\lambda_{0}\right)^{n}}
\end{array}
$$

for all $\lambda>\tilde{\|}\|V\|_{C_{b}^{2}\left(\mathbb{R}^{d}\right)}=\lambda_{0}$. Therefore the operator $A(t)-i V(t)$ generates a $C_{0}$ semigroup of contraction in $H^{2}\left(\mathbb{R}^{d}\right)$, with constants $\tilde{M}=1$ and $\tilde{\omega}=0$, hence the family of parts $\tilde{A}(t)-i \tilde{V}(t)$ is stable, as it is required in $H_{2}$.

The proofs of condition $H_{3}$ and of lemma 4.1 are easily deducible from the free case $(V \equiv 0)$, as the domain $H^{2}\left(\mathbb{R}^{d}\right)$ is equivalent the graph of the operator $A(t)$ and the solution can be written as:

$$
v\left(t ; 0, u_{0}\right)=U(t, 0) u_{0}+\int_{0}^{t} U(t, s) v\left(s ; 0, u_{0}\right) d s
$$

where $U(t, s)$ is generated by the $\{A(t), t \geq 0\}$.

We conclude with a remark: using the same technique it is possible to treat also an equation of the type $\left({ }^{6}\right)$ :

$$
\left\{\begin{array}{l}
i d u(t, x)+\frac{1}{2} \Delta u(t, x) d t-V(x) u(t, x) d t=\sum_{k=1}^{m} c_{k}(t, x) u(t, x) \circ d W_{k}(t) \\
u(0)=u_{0}
\end{array}\right.
$$

\footnotetext{
${ }^{6}$ This equation has been considered also in $[8,9]$.
} 
where $c_{k}$ are real regular functions.

In this case we transform it into a determistic family of equations evaluating the Itô formula for the product $u(t, x) \rho(t, x)$, where $\rho$ is the process defined as $\rho(t, x)=$ $\exp \left(i \sum_{k=1}^{m} c_{k}(s, x) \circ d W_{k}(s)\right)$. The flow $\rho$ defines a unitary operator $\Phi_{t} v(\cdot)=$ $v(\cdot) \rho(t, \cdot)$, and using the same approach we get results similar to those found in the case considered here.

\section{REFERENCES}

[1] F. Flandoli and H. Lisei. Conjugation of flows for spde's of parabolic type with multiplicative noise and some applications. Submitted.

[2] R. L. Hudson and K. R. Parthasarathy. Quantum Ito's formula and stochastic evolutions. Comm. Math. Phys., 93(3):301-323, 1984.

[3] H. Kunita. First order stochastic partial differential equations. In Stochastic analysis (Katata/Kyoto, 1982), pages 249-269. North-Holland, Amsterdam, 1984.

[4] R.M. Dudley, H. Kunita, and Ledrappier F. Ecole d'Été de Probabilités de Saint-Flour XII1982. Springer Verlag, Berlin, 1982.

[5] A. Pazy. Semigroups of linear operators and applications to partial differential equations. Springer-Verlag, New York, 1983.

[6] B. L. Rozovski1̌. Stochastic evolution systems. Kluwer Academic Publishers Group, Dordrecht, 1990.

[7] L. Tubaro. Some result on stochastic partial differential equations by the stochastic characteristics method. Stochastic Analysis and Applications, 6(2):217-230, 1988.

[8] A. Truman and T. Zastawniak. Stochastic PDE's of Schrödinger type and stochastic Mehler kernels - a path integral approach. In Seminar on Stochastic Analysis, Random Fields and Applications (Ascona, 1996), pages 275-282. Birkhäuser, Basel, 1999.

[9] A. Truman and T. Zastawniak. Stochastic Mehler kernels via oscillatory path integrals. $J$. Korean Math. Soc., 38(2):469-483, 2001.

[10] T. J. Zastawniak. Fresnel type path integral for the stochastic Schrödinger equation. Lett. Math. Phys., 41(1):93-99, 1997.

*Faculty of Mathematics and Computer Science, Babeş-Bolyai University, Str. Kogălniceanu Nr. 1, RO - 3400 Cluj-Napoca, Romania

E-MAIL: HANNE@MATH.UBBCLUJ.RO

** Dipartimento di Matematica, Politecnico di Milano, P.zza Leonardo da Vinci 32, 20133 Milano., Italy.

E-MAIL: GIUGUA@MATE.POLIMI.IT 